\title{
Enhanced Frequency Adaptive Demodulation Technique For Grid-Connected Converters
}

\author{
Hafiz Ahmed, Senior Member, IEEE, Samet Biricik, Senior Member, IEEE, Mohamed Benbouzid, \\ Fellow, IEEE
}

\begin{abstract}
This paper presents an enhanced frequency adaptive demodulation technique for grid-synchronization of grid-connected converters (GCC) in variable frequency condition. Demodulation works by generating demodulated voltages which contain undesired double frequency components. As a result, high-order low-pass filters (LPF) with high cutoff frequency are required to eliminate the undesired components. This reduces the dynamic performance. Frequency adaptive demodulation technique enhances the dynamic performance by rejecting the double frequency components as opposed to filtering, however, at the cost of additional computational complexity. This paper overcomes this problem by using double demodulation without recreating the double frequency component for rejection purpose. This reduces the computational complexity significantly. Suitability of proposed method is verified through numerical simulation and experimental study. Comparative study with existing frequency adaptive demodulation and second-order generalized integrator phase-locked loop (SOGI-PLL) techniques demonstrate the validity and performance improvement by the proposed technique.
\end{abstract} PLL.

Index Terms-Demodulation, Frequency Estimation,

\section{INTRODUCTION}

C LIMATE change is a serious concern of our time. In order to slow down the adverse effect of climate change, greenhouse gas emissions need to be reduced significantly. Approximately $60 \%$ of the global greenhouse gas emissions are linked to energy consumed by cities all over the world [1]. As such, sustainable and clean energy will play a major role to reduce the overall greenhouse gas emissions. In this context, renewable energy sources (RES) are considered to be a major player. RES are typically connected to the traditional power grid through grid-connected converters (GCC). To ensure maximum power transfer from RES to the grid, synchronized operation of the GCC is necessary. However, this require

Manuscript received XXX XX, 2020; revised XXX XX, 2020; accepted XXX XX, 2020. Date of publication XXX XX, 2020; date of current version $X X X X X, X X X X$. This work was supported in part by the Royal Society under grant RGSIR2\192245. Paper no. TIE-20-1509. (Corresponding author: Hafiz Ahmed.)

$\mathrm{H}$. Ahmed is with the School of Engineering and the Built Environment, Birmingham City University, Birmingham B4 7XG, United Kingdom (e-mail: hafiz.h.ahmed@ieee.org).

$\mathrm{S}$. Biricik is with the Department of Electrical and Electronic Engineering, European University of Lefke, Lefke 99728, Turkey, and also with the School of Electrical and Electronic Engineering, Technological University Dublin, Dublin D08 X622, Ireland.

M. Benbouzid is with the University of Brest, UMR CNRS 6027 IRDLs, 29238 Brest, France, and also with the Logistics Engineering College, Shanghai Maritime University, Shanghai 201306, China. accurate information of the grid voltage parameters. Grid voltage parameters are an integral part of the GCC controller [2]-[9]. This motivates the current work.

Estimating the parameters of grid voltage signals can be considered as an important technical problem. To address this challenging technical problem, many techniques have been proposed in the literature. In the ideal case, grid voltage is a periodic signal with known frequency. This makes frequency domain technique such as discrete Fourier transform (DFT) [10], [11] a suitable choice to estimate the parameters of grid voltage signal. Standard DFT has high memory and processing power requirement. To overcome this issue, recursive implementations are used in practice. In the presence of offnominal frequency, the performance of DFT suffers. This can be overcomed either by using variable sampling frequency or variable window length. However, variable sampling frequency is not suitable for control applications (issue with filter design) and variable window length can be computationally expensive. Regression-based techniques [12]-[14] are another popular technique available in the literature. This type of technique use a parameterized model of the grid voltage signal. This type of technique has a high memory requirement. Moreover, computationally expensive matrix inverse may also be required. This type of technique is often coupled with phase-locked loop (PLL) for the frequency estimation. As a result, PLL significantly influence the performance of regression-based techniques.

State-space observer [13], [15], [16] use a state-space model of the grid voltage as opposed to parameterized model used by regression-based techniques. State-space observer can either use fixed gain (e.g. Luenberger observer [15]) or variable gain (e.g. Kalman filter [13]). The performance of the Luenbergertype observer degrades heavily in the presence of disturbances (harmonics, measurement offset etc.). Kalman filter has better disturbance rejection capability (specially noise rejection), however, comes at the cost of high computational complexity. As the filter gain is continuously updated through realtime matrix inverse, it's implementation in low-cost microcontrollers can be limited.

Band-pass filter-type synchronization algorithms [17], [18] became very popular in recent times. Out of them, second order generalized integrator (SOGI) [18], [19] and adaptive notch filter (ANF) [17] are very similar in nature and uses frequency adaptive filter gain while self-tuning filter (STF) [20], [21] do not use any frequency adaptive gain. SOGI and ANF can be applied to both single and three-phase while STF is limited to three-phase. In phasor form, STF behaves 
as a complex first-order band-pass filter. In the state-space framework, the model of these filters resemble to that of a simple harmonic oscillator $(\mathrm{SHO})$ with eigenvalues $\pm i \omega$, where $\omega$ is the oscillation frequency. This model is based on ideal sinusoid. However, in practice, the grid voltage is not ideal. As such, in the presence of harmonic distortions, there will be modeling error. This modeling error will impact the estimation performance. One potential solution is to add additional filters (pre or in-loop). This comes at the cost of additional computational complexity and gains tuning.

Out of various techniques available in the literature, PLL [22]-[28] is probably the most popular technique used in the industrial applications. PLL is a closed-loop system and uses a linear phase detector (PD). Output of the PD is passed through a proportional-integral (PI)-type low-pass filter (LPF) to estimate the unknown grid frequency. Performance of the PLL depends heavily on the LPF tuning. The cutoff frequency of the LPF determines the dynamic response of the PLL. High bandwidth enhances the dynamic performance but at the cost of lower disturbance rejection capability. Open-loop techniques [29]-[31] present a solution to this problem by not requiring the feedback. Open-loop techniques generally use derivative to estimate the frequency. Numerical implementation of derivative can introduce estimation error at low sampling frequency. Moreover, noise can also deteriorate the performance of the derivative estimation and consequently the frequency estimation.

Demodulation uses idea originally developed for telecommunication application where received signals are mixed with known signals to extract the information (i.e. phase). Standard demodulation techniques (single-dimensional [32], [33] and multi-dimensional [34], [35]) mix the grid voltage signal (real or complex phasor) with sine and cosine signals of known frequency. As a result, two demodulated signals are obtained. Each signal is composed of a DC component containing the required information and a double frequency component. By using low-pass filter (LPF), the double frequency component can be easily removed. However, this requires a high-order LPF with high cutoff frequency e.g. between $f$ and $2 f$ with $f$ being the nominal frequency. As such, the dynamic performance can be comparatively slow. To overcome this issue, quadrature oscillator (QO)-based demodulation technique is used in [36]. This technique compensates the double frequency component through QO. This facilitates the use of low-order LPF with low cutoff frequency.

In this paper, we focus on demodulation technique. Our objective is to enhance the performance with low computational complexity. For this purpose, we propose the application of demodulation to three-phase system. First, we reduce the three-phase system to two-phase through Concordia transformation [37]. These two-phase signals are then demodulated with frequency adaptive sine and cosine signals. This helps to eliminate the double frequency component with low computational complexity. Performance of the proposed technique is compared with standard frequency adaptive demodulation [36] and second-order generalized integrator phase-locked loop (SOGI-PLL) [28] using various challenging test scenarios.

The rest of this paper is organized as follows: Sec. II

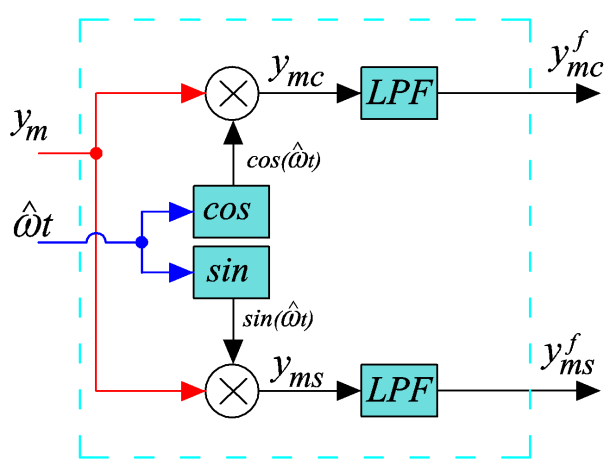

Figure 1. Block diagram of the standard demodulation technique.

summarizes the existing demodulation techniques while Sec. III describes the proposed development. Numerical simulation and experimental studies are given in Sec. IV. Finally, Sec. V concludes this paper.

\section{Demodulation Techniques: Short OVERView}

A single-phase grid voltage signal is generally modeled as a sine wave and given below:

$$
y_{m}(t)=A(t) \sin (\underbrace{\omega t+\phi(t)}_{\theta(t)})
$$

where the amplitude, frequency, phase and instantaneous phase are given by $A, \omega, \phi$, and $\theta$ respectively. The unknown frequency $\omega=\omega_{n}+\tilde{\omega}$, where $\omega_{n}=100 \pi$ is the nominal frequency and $\tilde{\omega}$ denotes the frequency deviation from the nominal frequency. Similarly, the instantaneous phase angle can be written as $\theta=\theta_{n}+\tilde{\theta}$ where $\theta_{n}=\omega_{n} t$ is the nominal instantaneous phase and $\tilde{\theta}=\tilde{\omega} t+\phi$ is the deviation from the nominal instantaneous phase. Demodulation technique works by generating demodulated voltages from the measured grid voltage signal (1). Basic overview of the standard demodulation technique is given in Fig. 1. For the purpose of demodulation, the grid voltage signal is multiplied with quadrature signals of known frequency. The demodulated voltages contain a double-frequency components. This can be easily rejected by a suitably designed low-pass filter (LPF). Performance of the demodulation technique relies heavily on the low-pass filtering. High rejection can only be achieved if low bandwidth is used to tune the filter. However, low bandwidth makes the dynamic response slow. Moreover, filter order plays a big role. Rejection efficiency increases with increasing order, however, comes at the cost of computational complexity. One solution to this problem could be frequency adaptive demodulation. Basic idea of frequency adaptive demodulation technique is given in Fig. 2. For the sake of conciseness, the frequency estimation block is avoided and has the same form as Fig. 3b. The main idea of this approach is to reject the double frequency components of standard demodulation. For this purpose quadrature oscillator (QO) is used in [36]. QO uses frequency adaptive quadrature signals to generate double frequency components. These double frequency components are added/subtracted with the demodulated voltages to get 


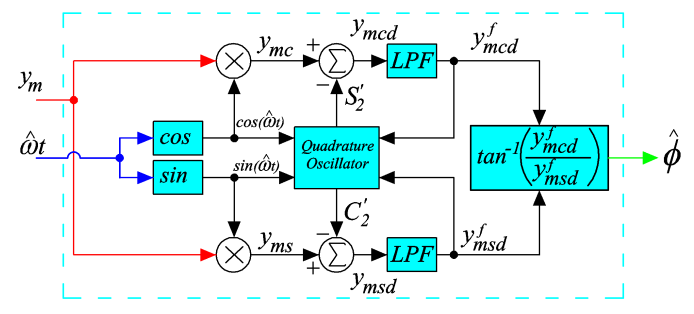

Figure 2. Block diagram of the frequency adaptive demodulation technique [36].

rid of the double frequency components. As a result, a loworder LPF with low cutoff frequency is sufficient to extract the desired signal as opposed to high-order and high cutoff frequency LPFs used in standard demodulation technique.

In the case of Frequency Adaptive Demodulation (FADM), the demodulated voltages are given by:

$$
\begin{aligned}
y_{m s} & =y_{m} \sin (\omega t) \\
& =\frac{A}{2} \cos (\phi)-\frac{A}{2}(\cos (2 \omega t+\phi)) \\
y_{m c} & =y_{m} \cos (\omega t) \\
& =\frac{A}{2} \sin (\phi)+\frac{A}{2}(\sin (2 \omega t+\phi))
\end{aligned}
$$

For further development, the modified demodulated voltages are denoted as $y_{m s d}$ and $y_{m c d}$ and the low-pass filtered version of the demodulated voltages are denoted by $y_{m s d}^{f}$ and $y_{m c d}^{f}$. The modified demodulated voltages are given by:

$$
\begin{aligned}
& y_{m s d}=y_{m s}-C_{2}^{\prime} \\
& y_{m c d}=y_{m c}-S_{2}^{\prime}
\end{aligned}
$$

where $C_{2}^{\prime}$ and $S_{2}^{\prime}$ are the outputs of the quadrature oscillator. The outputs are regenerated by mixing the filtered demodulated voltages $y_{m s d}^{f}$ and $y_{m c d}^{f}$ with $S_{2}=\sin (2 \hat{\omega} t)$ and $C_{2}=\cos (2 \hat{\omega} t)$. Finally, the outputs of the QO are given by:

$$
\begin{aligned}
& S_{2}^{\prime}=y_{m s d}^{f} S_{2}+y_{m c d}^{f} C_{2}=\frac{A}{2} \sin (2 \hat{\omega} t+\phi) \\
& C_{2}^{\prime}=-y_{m s d}^{f} C_{2}+y_{m c d}^{f} S_{2}=-\frac{A}{2} \cos (2 \hat{\omega} t+\phi)
\end{aligned}
$$

Thanks to the QO outputs (6) and (7), double frequency components can be rejected in eq. (2) and (3). However, this comes at the cost of additional computational complexity. As a result, there is scope to improve the computational complexity.

\section{Enhanced Frequency Adaptive Demodulation}

Balanced three phase voltages are given by:

$$
\begin{aligned}
& y_{a}=A(t) \cos (\omega t+\phi(t)) \\
& y_{b}=A(t) \cos (\omega t+\phi(t)-2 \pi / 3) \\
& y_{c}=A(t) \cos (\omega t+\phi(t)+2 \pi / 3)
\end{aligned}
$$

By applying Concordia transformation (CT) [37], the threephase voltages $y_{a b c}=\left[\begin{array}{lll}y_{a} & y_{b} & y_{c}\end{array}\right]^{T}$ can be transformed into two phase voltages using the following formula [37]:

$$
y^{(c)}=\left[\begin{array}{l}
y_{1}^{(c)} \\
y_{2}^{(c)}
\end{array}\right]=C y_{a b c}
$$

where the CT matrix is given by:

$$
C=\sqrt{\frac{2}{3}}\left[\begin{array}{ccc}
\sqrt{\frac{2}{3}} & -\frac{1}{\sqrt{6}} & -\frac{1}{\sqrt{6}} \\
0 & \frac{1}{\sqrt{2}} & -\frac{1}{\sqrt{2}}
\end{array}\right] .
$$

From eq. (11), the individual phase voltages are obtained as:

$$
\begin{aligned}
& y_{1}=A \cos (\omega t+\phi) \\
& y_{2}=A \sin (\omega t+\phi)
\end{aligned}
$$

By mixing frequency adaptive quadrature signals with signal $y_{1}$, the demodulated voltages can be obtained as:

$$
\begin{aligned}
y_{1 s d} & =y_{1} \sin (\hat{\omega} t) \\
& =\frac{A}{2}[\sin \{(\omega+\hat{\omega}) t+\phi\}-\sin \{(\omega-\hat{\omega}) t+\phi\}] \\
& \approx \frac{A}{2}\{\sin (2 \omega t+\phi)-\sin (\phi)\} \\
y_{1 c d} & =y_{1} \cos (\hat{\omega} t) \\
& =\frac{A}{2}[\cos \{(\omega-\hat{\omega}) t+\phi\}+\cos \{(\omega+\hat{\omega}) t+\phi\}] \\
& \approx \frac{A}{2}\{\cos (\phi)+\cos (2 \omega t+\phi)\}
\end{aligned}
$$

In obtaining eq. (15) and (16), we have assumed quasi-locked condition i.e. $\omega \approx \hat{\omega}$. Similar to the above approach, by mixing frequency adaptive quadrature signals with signal $y_{2}$, the demodulated voltages can be obtained as:

$$
\begin{aligned}
y_{2 s d} & =y_{2} \sin (\hat{\omega} t) \\
& =\frac{A}{2}[\cos \{(\omega-\hat{\omega}) t+\phi\}-\cos \{(\omega+\hat{\omega}) t+\phi\}] \\
& \approx \frac{A}{2}\{\cos (\phi)-\cos (2 \omega t+\phi)\} \\
y_{2 c d} & =y_{2} \cos (\hat{\omega} t) \\
& =\frac{A}{2}[\sin \{(\omega+\hat{\omega}) t+\phi\}+\sin \{(\omega-\hat{\omega}) t+\phi\}] \\
& \approx \frac{A}{2}\{\sin (2 \omega t+\phi)+\sin (\phi)\}
\end{aligned}
$$

From the demodulated voltages (15)-(18), the following DC signals can be obtained:

$$
\begin{aligned}
& x_{\phi}=y_{1 c d}+y_{2 s d} \approx A \cos (\phi) \\
& y_{\phi}=y_{2 c d}-y_{1 s d} \approx A \sin (\phi)
\end{aligned}
$$

From eq. (19) and (20), it is clear that the proposed technique does not require low-pass filter to generate the DC signals 


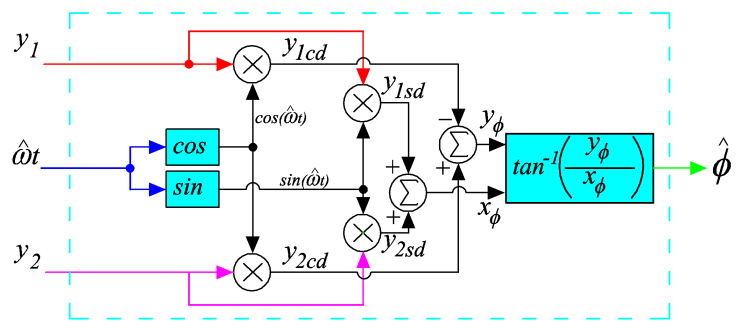

(a)

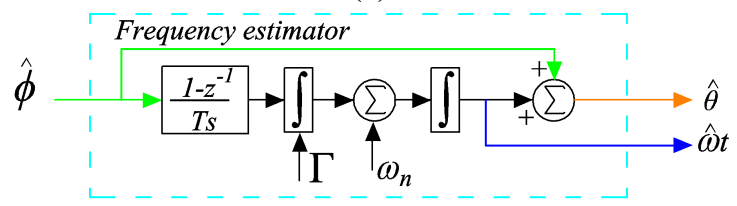

(b)

Figure 3. Block diagram of the proposed enhanced demodulation technique suitable for three-phase system: a) enhanced demodulation, b) frequency estimation.

using demodulation unlike the standard and frequency adaptive demodulation. This is a clear advantage of the proposed technique w.r.t. existing literature. However, by adding loworder low cutoff frequency low-pass filters, disturbance rejection capability of the proposed technique can be enhanced. From the DC signals, the amplitude and phase angle can be estimated as:

$$
\begin{aligned}
\hat{A} & =\sqrt{x_{\phi}^{2}+y_{\phi}^{2}} \\
\hat{\phi} & =\arctan \left(y_{\phi} / x_{\phi}\right)
\end{aligned}
$$

Proposed technique requires an estimate of the grid frequency $\omega$. This can be estimated from the estimated phase-angle $\hat{\phi}$. For this purpose, we consider the idea used in [14], [38]. In this approach, time derivative of the phase angle can be considered as an approximation of the frequency deviation dynamics. Whenever the signal frequency changes, estimation of $\phi$ also enters into the transient regime. As a result, in the transient state, $\phi$ also contains deviation from the nominal frequency information. By integrating the nominal frequency deviation dynamics, the actual deviation can be calculated. To tune the convergence of the frequency deviation, a positive gain $\Gamma$ for the integrator can be used. Block diagram of the proposed enhanced demodulation technique is given in Fig. 3.

The proposed technique is motivated by the FADM [36] described in Sec. II. Proposed technique reduces the multiplication and addition/subtraction operation of FADM by two times (cf. Sec. IV for details). In addition to the computational complexity reduction, the proposed technique has a smoother response compared to FADM. FADM estimate the double frequency components and reject it. Since complete rejection is not possible in practice, high-order low-pass filter is required for steep rollover. In the proposed case, since direct rejection is considered, no low-pass filter or low-order low-pass filter is sufficient to eliminate any undesirable frequency components. High-order low-pass filter means more design parameters lead-

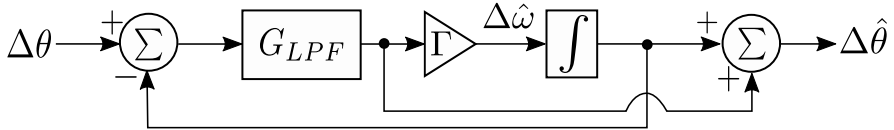

(a)

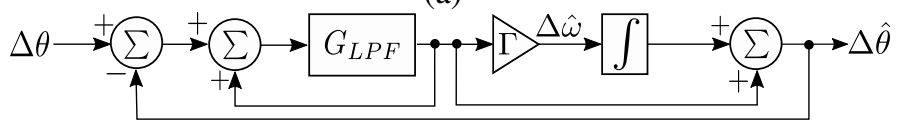

(b)

Figure 4. Mathematical model of the proposed technique, (a) smallsignal model and (b) alternative feedback representation of the smallsignal model.

ing to implementation and tuning complexities. In summary, computational and design complexity reduction and smooth response are the main advantages of the proposed technique compared to FADM.

Proposed technique do not use any orthogonal signal generator. Using basic trigonometric operations together with firstorder differentiation and integration for frequency estimation. These are less sensitive to sampling frequency. In contrast, SOGI for example typically use third order Adams-Bashforth discretization for the continuous integrators. As such, the performance degrades in the low sampling frequencies. In this context, relative insensitivity to sampling frequency can also be considered as an advantage of the proposed technique.

\section{A. Small-Signal Modeling and Parameter Tuning}

The proposed enhanced demodulation technique works as a phase angle detector. For the phase angle extraction purpose, instantaneous phase can be written as, $\theta=\theta_{n}+\Delta \theta$, where $\theta_{n}=\omega_{n} t$ is the nominal instantaneous phase and $\Delta \theta=\Delta \omega+\phi$ is the deviation from the nominal value. The proposed technique extracts the phase angle $\phi$ and estimate the frequency from the estimated phase angle. Then from the block diagram of the proposed technique given in Fig. 3, the small-signal model of the proposed technique can be easily obtained and given in Fig. 4. In this model, $G_{L P F}(s)$ is the transfer function of the LPF and given by $\omega_{c} /\left(s+\omega_{c}\right)$, where $\omega_{c}$ is the cutoff frequency of the filter. To validate the model, let us consider that $\omega_{c}=\omega_{n} / 3 \mathrm{rad} / \mathrm{sec}$. and $\Gamma=50$. Validation of the model is given in Fig. 5. Results in this figure show that the model is very accurate when the phase angle had a $+45^{\circ}$ step change in the phase angle.

From the alternative feedback representation of the smallsignal model given in Fig. 4b, the open-loop transfer function of the proposed technique is given by:

$$
G_{\mathrm{OL}}(s)=\frac{G_{L P F}(s)}{1-G_{L P F}(s)} \frac{s+\Gamma}{s}
$$

From the open-loop transfer function, the closed-loop transfer function of the proposed technique is given by:

$$
\begin{aligned}
\frac{\Delta \hat{\theta}}{\Delta \theta}(s) & =\frac{G_{\mathrm{OL}}(s)}{1+G_{\mathrm{OL}}(s)} \\
& =\frac{\omega_{c} s+\Gamma \omega_{c}}{s^{2}+\omega_{c} s+\Gamma \omega_{c}}
\end{aligned}
$$




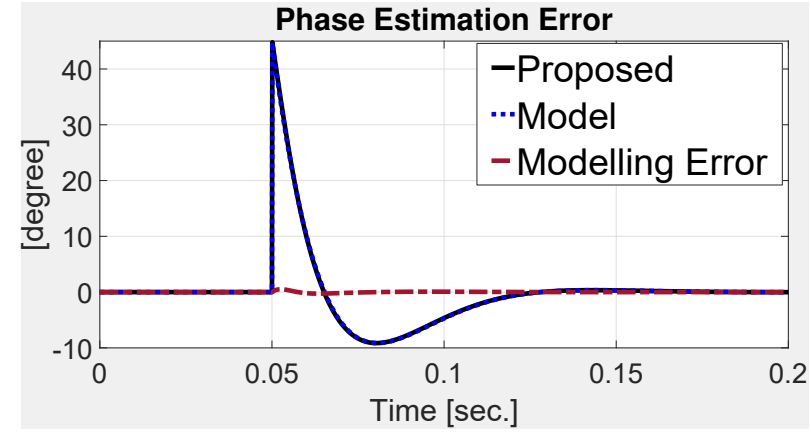

Figure 5. Validation of the small-signal model for the proposed technique.

Table I

Number of MATHEMATICAL OPERATIONS INVOLVED IN THE IMPLEMENTATION OF THE DEMODULATION TECHNIQUES

\begin{tabular}{cccccc}
\hline \hline & $\times$ & \pm & IT & TG & LPF \\
\hline FADM & 11 & 5 & 1 & 2 & 2 \\
\hline Proposed & 6 & 2 & 1 & 2 & 2 \\
IT-Inverse Tangent, TG- Trigonometric & operation
\end{tabular}

By comparing the denominator polynomial of the closedloop transfer function (24) with that of the standard secondorder transfer function denominator i.e. $s^{2}+2 \zeta \omega_{0} s+\omega_{0}^{2}$, the following formulas can be obtained for the tuning parameters $\omega_{c}$ and $\Gamma$ :

$$
\Gamma=\frac{\omega_{0}^{2}}{\omega_{c}}, \zeta=\frac{\omega_{c}}{2 \omega_{0}}
$$

From the tuning formula (25), just by selecting the LPFs cutoff frequency $\omega_{c}$ and the damping coefficient $\zeta$, the frequency estimation gain $\Gamma$ can be easily calculated. For a $\omega_{c}=\omega_{n} / 3 \mathrm{rad} / \mathrm{sec}$. and $\zeta=1 / \sqrt{2}, \Gamma=52$ can be found.

\section{Results and Discussions}

In this Section, numerical simulation and experimental study will be considered to demonstrate the suitability of the proposed enhanced demodulation technique. As comparison methods, frequency adaptive demodulation (FADM) (Sec. II and [36]) and SOGI-based PLL for three-phase system [28] have been considered. To implement FADM and the proposed technique, two low-pass filter of order 1 with cutoff frequency $\omega_{n} / 3 \mathrm{rad} / \mathrm{sec}$. are considered. Moreover, the frequency estimation parameter has been chosen as $\Gamma=50$. Gains of the SOGI-PLL are selected as $k=\sqrt{2}, k_{p}=222$ and $k_{i}=6169$ as suggested in [28]. Number of mathematical operations required to implement the proposed and the frequency adaptive demodulation technique are given in Table I. Table I shows that the proposed technique reduces the number of multiplication and summation operation by order of two. As such the proposed technique can be considered significantly simpler than the existing demodulation technique. It is to be noted here that the demodulation techniques including the proposed one use arctan function. Standard implementation of arctanfunction can be computationally expensive for microcontroller-based

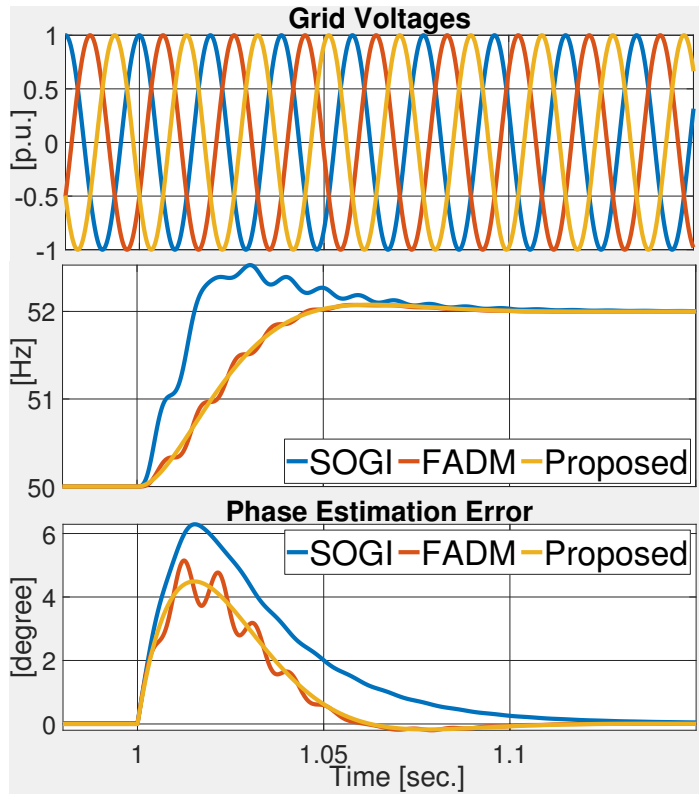

Figure 6. Numerical simulation results for Test-I: $+2 \mathrm{~Hz}$ change in frequency.

practical implementation. Coordinate Rotation Digital Computer (CORDIC) [39] approximation of arctan can significantly reduce the computational complexity for practical implementation. In this work, C2000 series microcontrollers from Texas Instruments is used for practical implementation. The IQmath library [40] for C2000 series supports CORDIC implementation of arctan and used in our work.

\section{A. Simulation Study}

In this Section, Matlab/Simulink-based simulation studies are considered. All the techniques have been implemented in Simulink with a sampling frequency $10 \mathrm{kHz}$. To test the performance of the algorithms, the following tests are considered:

- Test-I: $+2 \mathrm{~Hz}$ change in frequency

- Test-II: $+45^{\circ}$ change in phase

- Test-III: Harmonics robustness study

- Test-IV: +0.1 p.u. DC offset in phase $a$.

Numerical simulation results for Test-I are given in Fig. 6. Test results as shown in Fig. 6 reveals that all the techniques can successfully track the change in frequency. FADM and the proposed technique are similar in nature and almost identical performance can be observed. However, it is to be noted here that the proposed technique has a very smooth response as opposed to FADM. FADM estimates the double frequency components and reject it by taking the opposite sign. Whenever any parameters in the grid voltage change, the FADM estimator enters into transient state. This contributes to the oscillatory response of FADM. In the proposed approach, the double frequency components are directly rejected. This leads to a smother response compared to FADM. The peak phase estimation error overshoot is $\approx 1^{\circ}$ less for the proposed technique. Unlike the demodulation techniques, SOGI-PLL has a relatively slow response w.r.t. the change in frequency. 


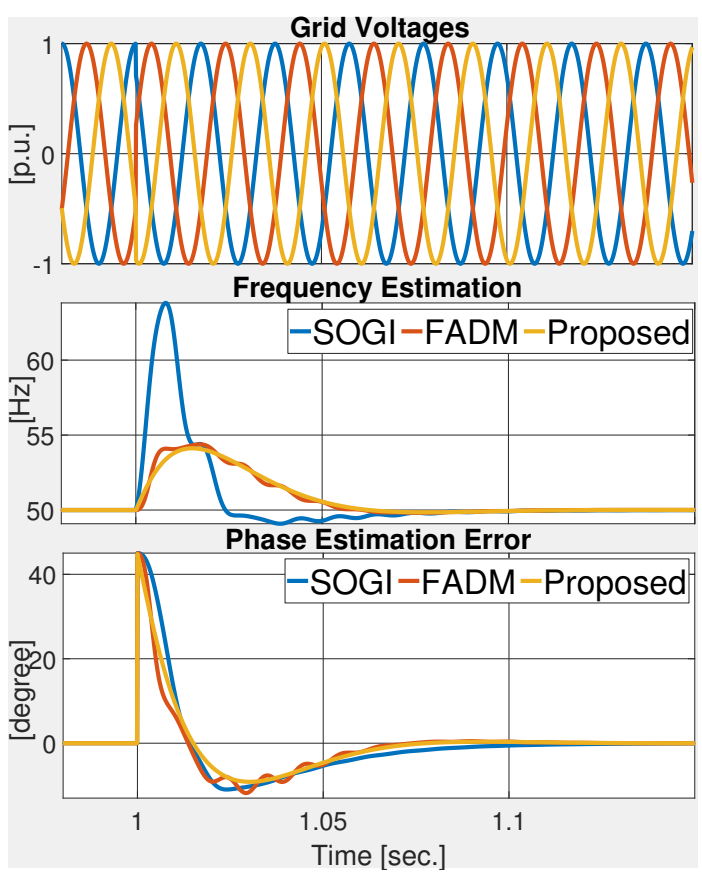

Figure 7. Numerical simulation results for Test-II: $+45^{\circ}$ change in phase.

In addition, the peak phase estimation error overshoot is also higher.

Sudden change in the grid phase is a challenging scenario for grid-synchronization techniques. Test-II considers that the grid suddenly experience a $+45^{\circ}$ phase change. Numerical simulation results for this test can be seen in Fig. 7. All the techniques performed similar to Test-I. FADM and the proposed technique were very quick to detect the change in phase and estimated the frequency and instantaneous phase within few cycles. The comparative techniques have very similar peak overshoot value for the frequency estimation. In case of phase estimation error, proposed technique's convergence time is very similar to FADM, however, a very smooth secondorder response can be observed unlike FADM.

The presence of harmonics can often be unavoidable. As such, grid-synchronization algorithms need to be harmonic robust. To test the harmonic robustness of the considered techniques, test-III considers a lightly distorted grid. The harmonic grid voltage components are: $3^{\text {rd }}-1.1 \%, 5^{\text {th }}-2.8 \%, 7^{\text {th }}-1.4 \%$, $9^{\text {th }}-2.3 \%, 11^{\text {th }}-1.5 \%, 30 \mathrm{~Hz}$ sub-harmonics $-1.1 \%$, and $180 \mathrm{~Hz}$ inter-harmonics $-1.3 \%$. Simulation results for test-III are given in Fig. 8. When the grid switches from undistorted to distorted mode, all the techniques show estimation ripple. However, out of the three methods, the proposed technique has the lowest estimation ripple. In the case of phase estimation error, FADM and the proposed technique have very similar performance while SOGI-PLLs estimation ripple is significantly higher than the other two techniques. Results in Fig. 8 show the suitability of the proposed technique even in distorted grid.

DC offset is another issue that can present significant challenge to grid-synchronization algorithms. DC offset may arises from the conversion of analog voltage signal to digital signal
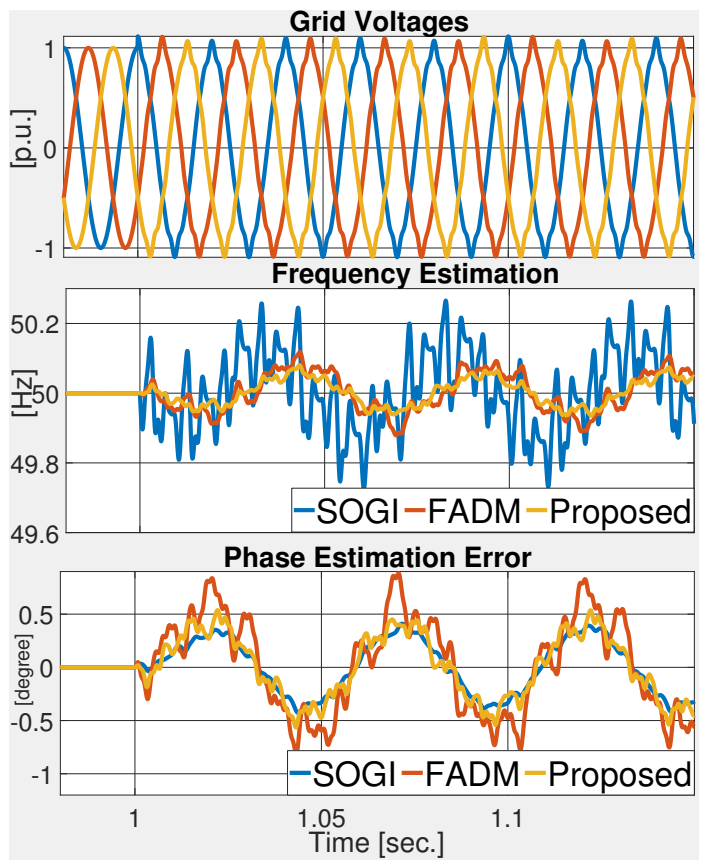

Figure 8. Numerical simulation results for test-III: harmonic robustness study.
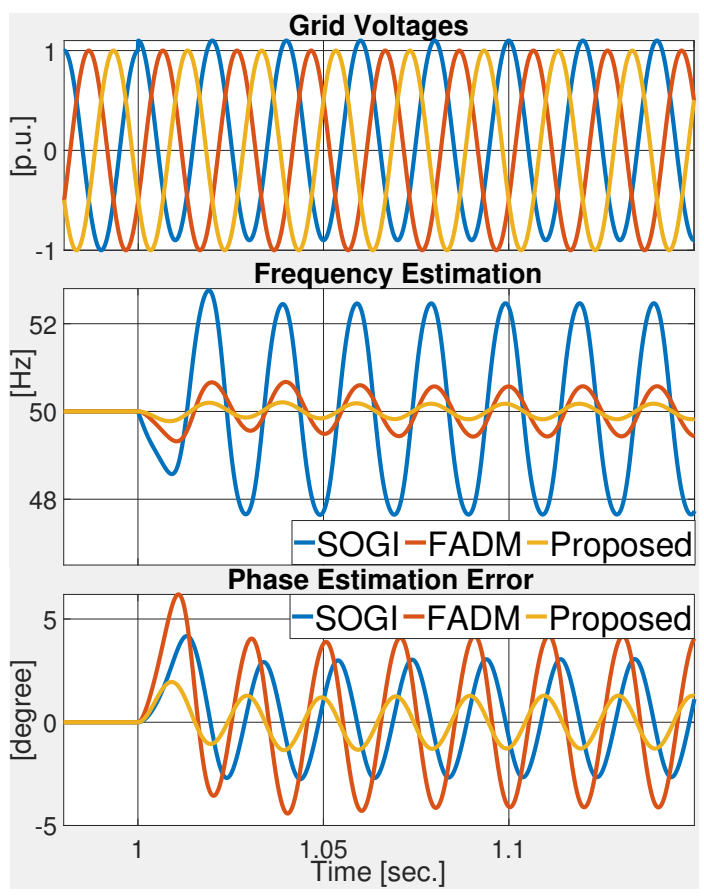

Figure 9. Simulation results for test-IV: +0.1 p.u. DC offset in phase $a$. 
to be used inside micro-controller for control and monitoring purpose. Test-IV considers a DC offset of +0.1 p.u. in the phase $a$ of the three-phase voltage signals. Simulation results for this case are given in Fig. 9. These figures reveal that the presence of DC offset effects the performance of the all the considered techniques. Out of three techniques, the proposed technique has the lowest ripple magnitude for the frequency estimation. The ripple magnitude of FADM and SOGI-PLL are few times higher with respect to the proposed technique. In the case of phase estimation error, similar to testIII, proposed technique has the lowest ripple magnitude while the performance of SOGI-PLL and FADM are significantly worse than that of the proposed technique.

Numerical simulation results as presented in Figs. 6-9 demonstrate that despite being computationally simpler than FADM, proposed technique performed either similar/better than FADM and SOGI-PLL. This shows that effectiveness of the proposed technique over similar literature.

\section{B. Experimental Study}

The experimental platform shown in Fig. 10 is used to investigate the performance of the studied algorithms. The platform is designed using a digital signal processor TMS320F28379D and associated measurement circuit. Three LEM LV 25-P Hall effect transducers are used to measure the phase voltages at the PCC (point of common coupling). The studied algorithms are modeled in Matlab/Simulink and integrated into the DSP using Code Composer Studio ${ }^{\mathrm{TM}}$ (CCStudio) through code generation. Six experimental tests are considered and they are: voltage sag and swell, frequency drop and jump, harmonics, and DC offset. To observe the effects of voltage harmonics on the algorithms, a non-linear load is used to draw harmonically distorted currents. The consumed distorted currents generate a voltage drop on the line inductors and this action creates harmonic distortion on the PCC voltages. Frequency variation is obtained by using a synchronous generator where the speed of the machine is controlled to obtain the frequency variations.

In the first two tests, $\pm 2 \mathrm{~Hz}$ frequency change are considered. Comparative experimental results for this test are given in Figures 11 and 12. Experimental results show that the proposed technique has good convergence speed compared to the other techniques. Moreover, the peak overshoot is also within acceptable limit for the proposed technique. The numerical simulation and experimental results have close match.

In the third and fourth tests, voltage sag and swell are considered and the experimental results are given in Figures 13 and 14. These figures show that the proposed technique is very insensitive to voltage sag/swell. In both cases, the frequency variation in the transient state was between $\pm 0.03 \mathrm{~Hz}$. This demonstrates the suitability of the proposed technique in the presence of voltage sag/swell.

Experimental results for harmonic distortion case are given in Fig. 15. Results show that the proposed technique can robustly estimate the frequency even in heavily distorted grid. Moreover, the proposed technique also has the lowest estimation ripple among the comparative methods. Similar performance can be observed for DC offset case also (cf.

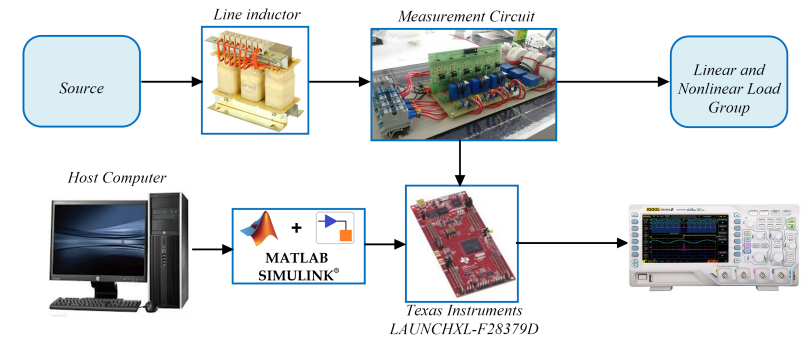

Figure 10. Overview of the considered experimental setup.

Fig. 16). These results show that the proposed technique is less sensitive to grid abnormalities such as distortion and DC offset.

All the experimental results given in this section show that the proposed technique is very suitable for practical implementation.

\section{CONCLUSION AND Future WORKS}

An enhanced demodulation technique is proposed in this paper for the synchronization of grid-connected converters. The proposed technique enhances the performance of existing frequency adaptive demodulation technique using lower computational complexity. This is a significant advantage of the proposed technique. Using various scenarios, including frequency and phase variations, harmonic distortions (odd, inter, and sub-harmonics), and DC offset, the performance of the proposed technique is evaluated and compared with two other advanced techniques. Results demonstrated that the proposed technique has fast convergence and good disturbance rejection capability. To further enhance the harmonic disturbance rejection property of the proposed technique, Kalman filter-based pre-loop filter will be considered as a future work.

\section{REFERENCES}

[1] U. Habitat, "Cities and pollution contribute to climate change," 2019 (accessed June 29, 2020). [Online]. Available: https://www.un.org/en/climatechange/cities-pollution.shtml

[2] F. Chishti, S. Murshid, and B. Singh, "Frequency adaptive multistage harmonic oscillator for renewable based microgrid under non-ideal grid conditions," IEEE Transactions on Industrial Electronics, pp. 1-1, 2020.

[3] — , "Weak grid intertie WEGS with hybrid generalized integrator for power quality improvement," IEEE Transactions on Industrial Electronics, vol. 67, no. 2, pp. 1113-1123, 2020.

[4] A. Rahoui, A. Bechouche, H. Seddiki, and D. O. Abdeslam, "Virtual flux estimation for sensorless predictive control of PWM rectifiers under unbalanced and distorted grid conditions," IEEE Journal of Emerging and Selected Topics in Power Electronics, pp. 1-1, 2020.

[5] M. Merai, M. W. Naouar, I. Slama-Belkhodja, and E. Monmasson, "An adaptive PI controller design for DC-link voltage control of single-phase grid-connected converters," IEEE Transactions on Industrial Electronics, vol. 66, no. 8, pp. 6241-6249, 2019.

[6] A. Rahoui, A. Bechouche, H. Seddiki, and D. O. Abdeslam, "Grid voltages estimation for three-phase PWM rectifiers control without AC voltage sensors," IEEE Transactions on Power Electronics, vol. 33, no. 1, pp. 859-875, 2018.

[7] B. Lekouaghet, A. Boukabou, N. Lourci, and K. Bedrine, "Control of PV grid connected systems using MPC technique and different inverter configuration models," Electric Power Systems Research, vol. 154, pp. 287-298, 2018.

[8] A. Safa, E. M. Berkouk, Y. Messlem, and A. Gouichiche, "A robust control algorithm for a multifunctional grid tied inverter to enhance the power quality of a microgrid under unbalanced conditions," International Journal of Electrical Power \& Energy Systems, vol. 100, pp. 253-264, 2018. 


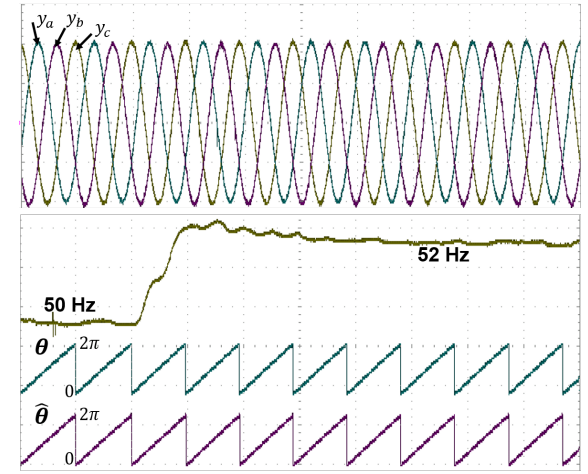

a) SOGI-PLL

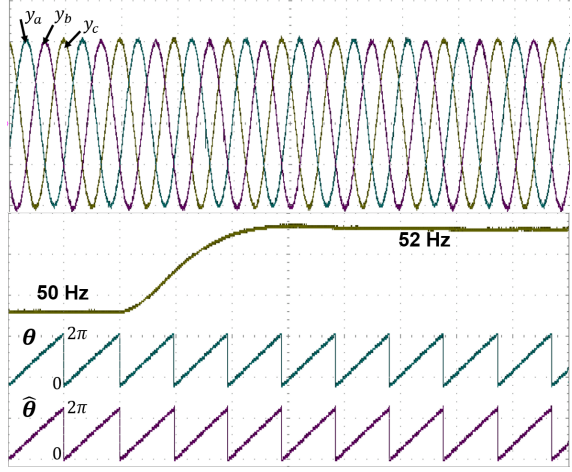

b) FADM

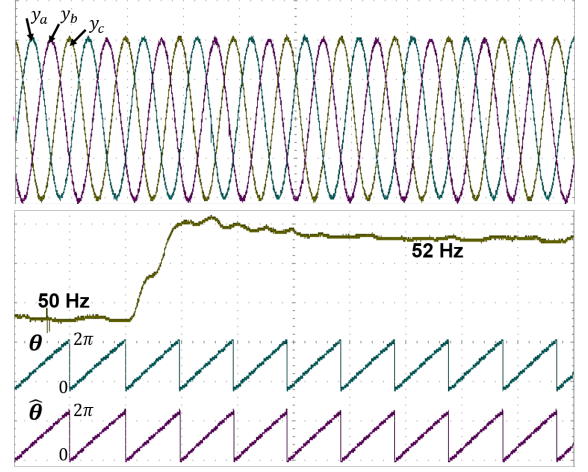

c) Proposed Technique

Figure 11. Experimental results for $+2 \mathrm{~Hz}$ frequency jump: a) SOGI-PLL, b) FADM, and c) proposed technique.

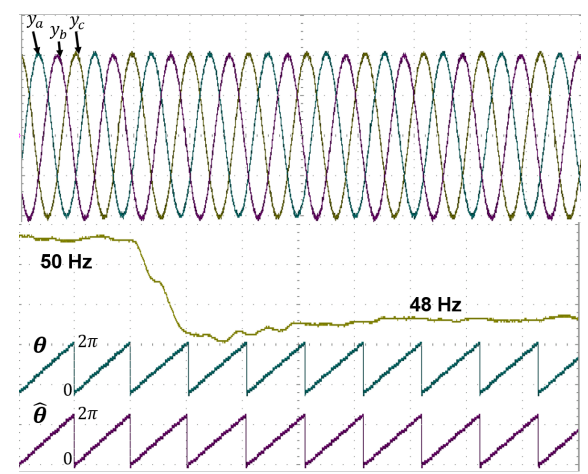

a) SOGI-PLL

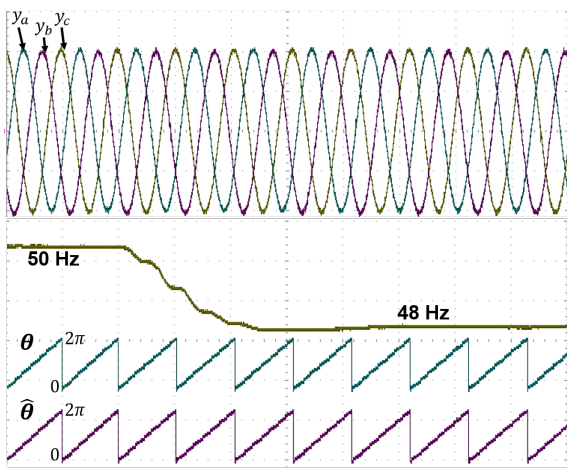

b) FADM

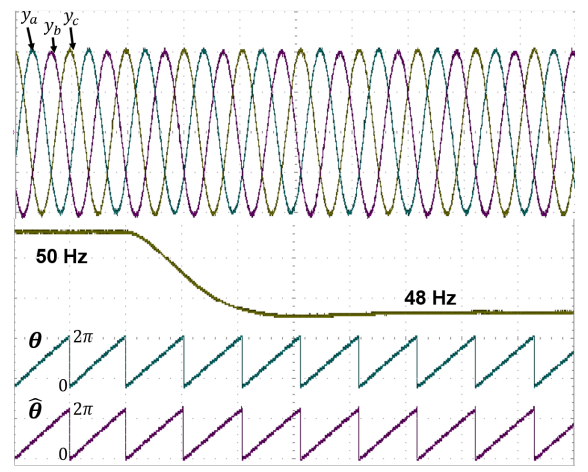

c) Proposed Technique

Figure 12. Experimental results for $-2 \mathrm{~Hz}$ frequency drop: a) SOGI-PLL, b) FADM, and c) proposed technique.

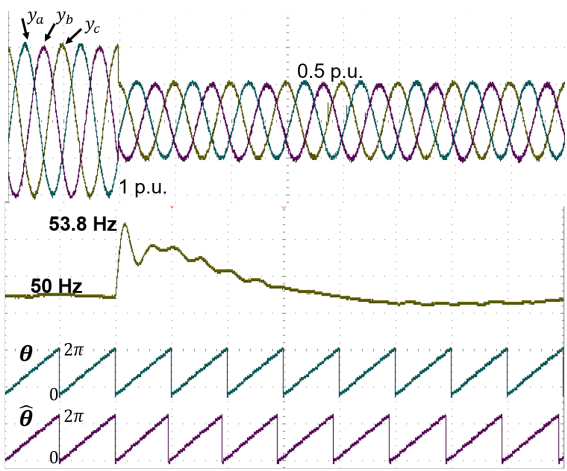

a) SOGI-PLL

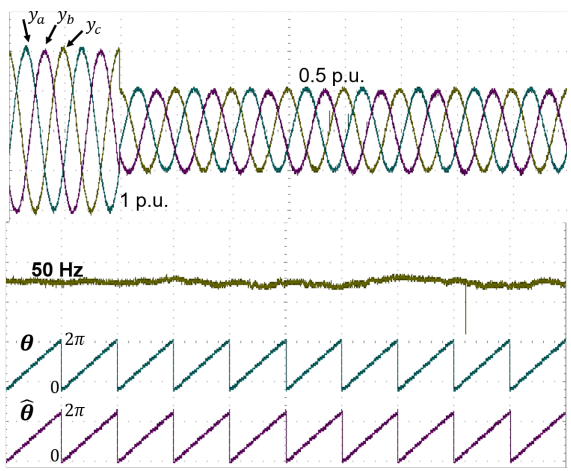

b) FADM

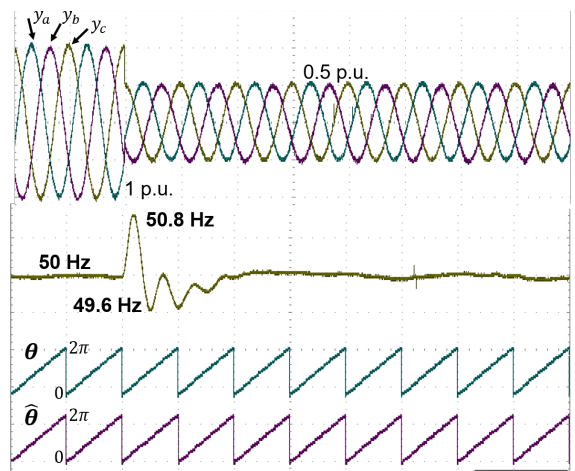

c) Proposed Technique

Figure 13. Experimental results for -0.5 p.u. voltage sag: a) SOGI-PLL, b) FADM, and c) proposed technique.

[9] H. Ahmed, S.-A. Amamra, and M. Bierhoff, "Frequency-locked loopbased estimation of single-phase grid voltage parameters," IEEE Transactions on Industrial Electronics, vol. 66, no. 11, pp. 8856-8859, 2019.

[10] T. Tyagi and P. Sumathi, "Comprehensive performance evaluation of computationally efficient discrete Fourier transforms for frequency estimation," IEEE Transactions on Instrumentation and Measurement, vol. 69 , no. 5, pp. 2155-2163, 2020.

[11] B. P. McGrath, D. G. Holmes, and J. J. H. Galloway, "Power converter line synchronization using a discrete Fourier transform (DFT) based on a variable sample rate," IEEE Transactions on Power Electronics, vol. 20, no. 4, pp. 877-884, 2005.

[12] Y. Terriche, J. M. Guerrero, and J. C. Vasquez, "Performance improvement of shunt active power filter based on non-linear least-square approach," Electric Power Systems Research, vol. 160, pp. 44-55, 2018.

[13] Y. Amirat, Z. Oubrahim, H. Ahmed, M. Benbouzid, and T. Wang,
"Phasor estimation for grid power monitoring: Least square vs linear Kalman filter," Energies, vol. 13, no. 10, p. 2456, 2020.

[14] H. Ahmed, I. Salgado, I. Chairez, and M. Benbouzid, "Robust gradient estimator for unknown frequency estimation in noisy environment: Application to grid-synchronization," IEEE Access, vol. 8, pp. 70693 $70702,2020$.

[15] H. Ahmed, S.-A. Amamra, and I. Salgado, "Fast estimation of phase and frequency for single-phase grid signal," IEEE Transactions on Industrial Electronics, vol. 66, no. 8, pp. 6408-6411, 2019.

[16] H. Ahmed, M. Benbouzid, M. Ahsan, A. Albarbar, and M. Shahjalal, "Frequency adaptive parameter estimation of unbalanced and distorted power grid," IEEE Access, vol. 8, pp. 8512-8519, 2020.

[17] S. Biricik, H. Komurcugil, N. D. Tuyen, and M. Basu, "Protection of sensitive loads using sliding mode controlled three-phase DVR with adaptive notch filter," IEEE Transactions on Industrial Electronics, 


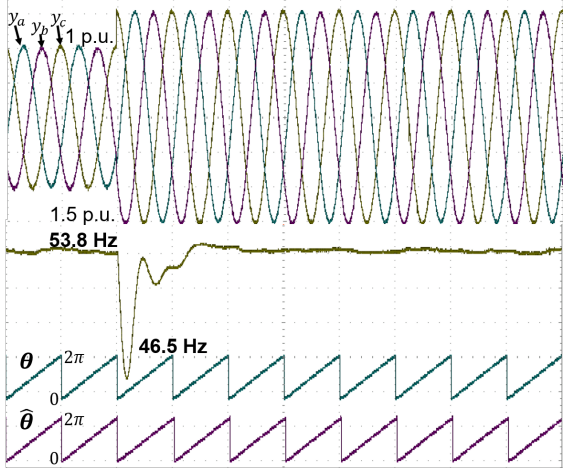

a) SOGI-PLL

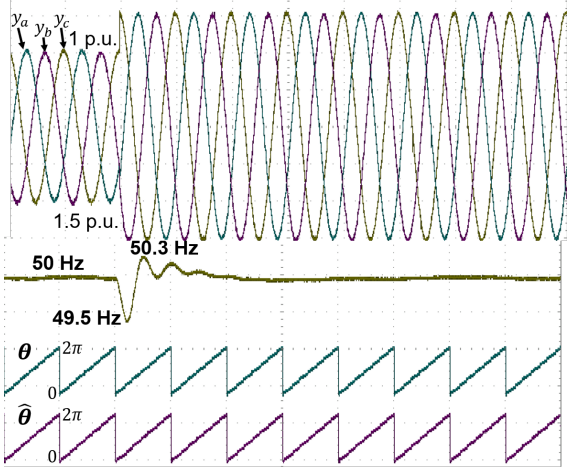

b) FADM

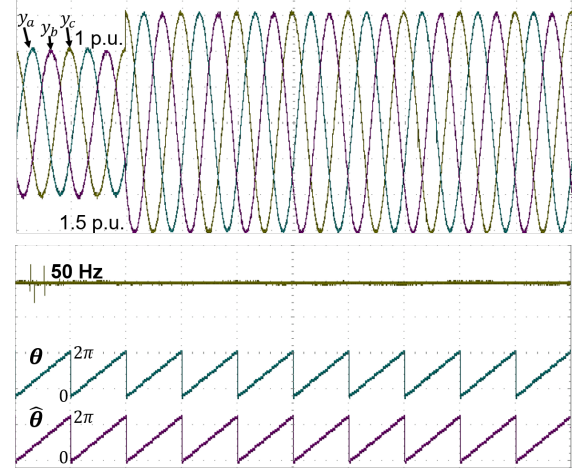

c) Proposed Technique

Figure 14. Experimental results for +0.5 p.u. voltage swell: a) SOGI-PLL, b) FADM, and c) proposed technique.
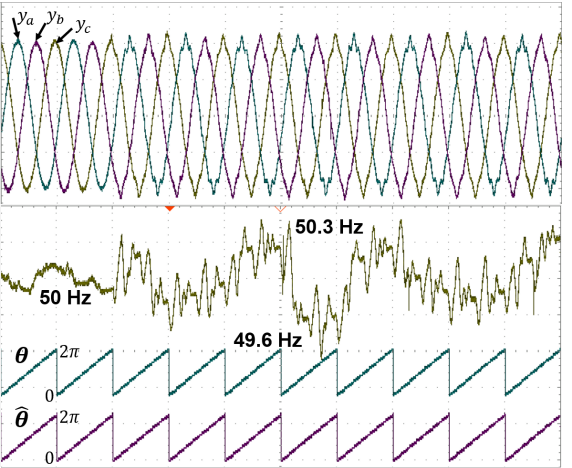

a) SOGI-PLL

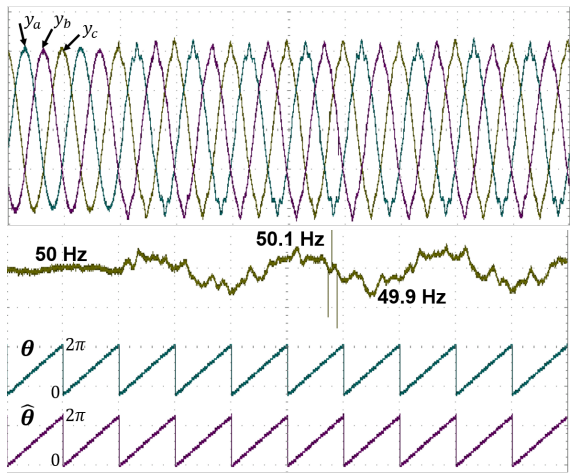

b) FADM

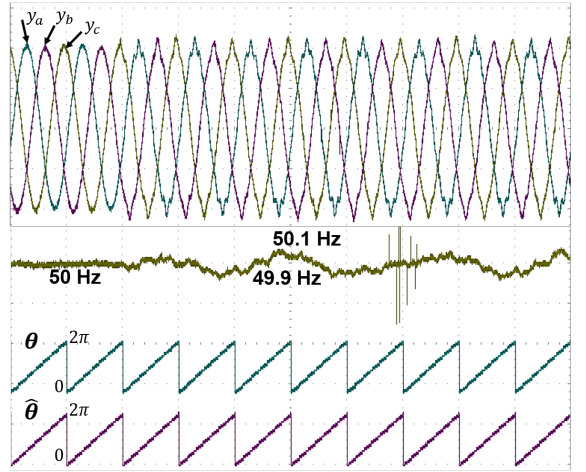

c) Proposed Technique

Figure 15. Experimental results for harmonic disturbance: a) SOGI-PLL, b) FADM, and c) proposed technique.

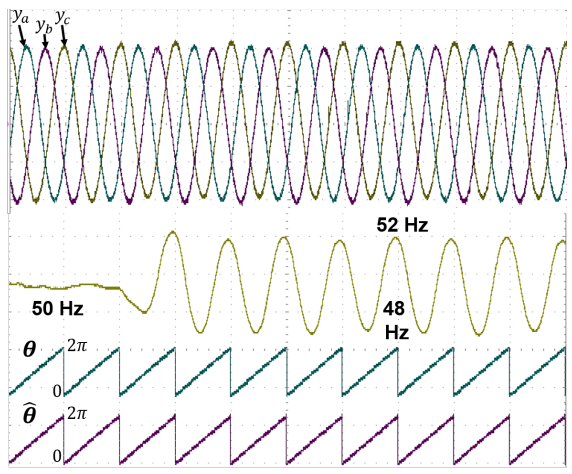

a) SOGI-PLL

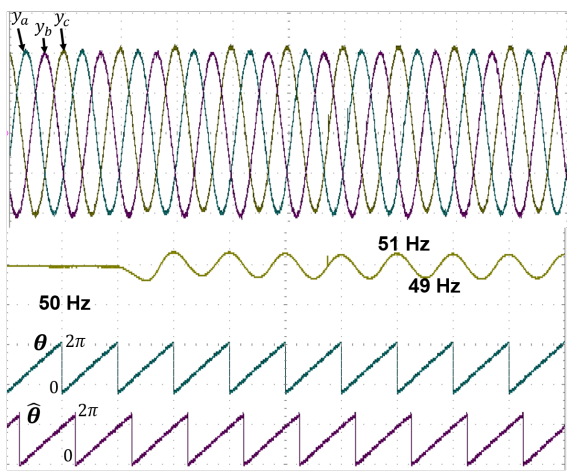

b) FADM

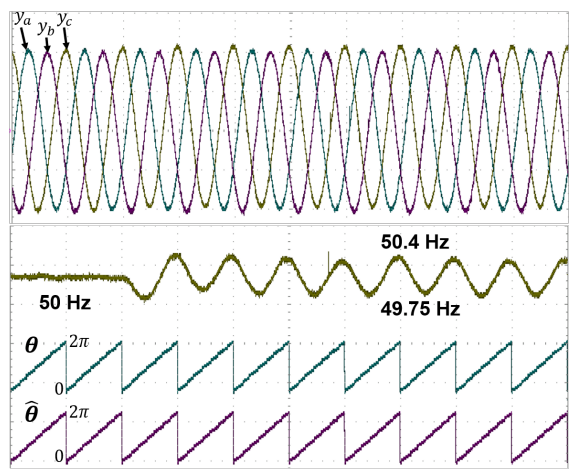

c) Proposed Technique

Figure 16. Experimental results for +0.05 p.u. DC offset in phase $a$ : a) SOGI-PLL, b) FADM, and c) proposed technique.

vol. 66, no. 7, pp. 5465-5475, 2018.

[18] A. Kherbachi, A. Chouder, A. Bendib, K. Kara, and S. Barkat, "Enhanced structure of second-order generalized integrator frequencylocked loop suitable for DC-offset rejection in single-phase systems," Electric Power Systems Research, vol. 170, pp. 348-357, 2019.

[19] P. Rodríguez, A. Luna, I. Candela, R. Mujal, R. Teodorescu, and F. Blaabjerg, "Multiresonant frequency-locked loop for grid synchronization of power converters under distorted grid conditions," IEEE Transactions on Industrial Electronics, vol. 58, no. 1, pp. 127-138, 2011.

[20] Y. Hoon, M. A. M. Radzi, M. K. Hassan, and N. F. Mailah, "Operation of three-level inverter-based shunt active power filter under nonideal grid voltage conditions with dual fundamental component extraction," IEEE Transactions on Power Electronics, vol. 33, no. 9, pp. 7558-7570, 2017.

[21] T. Ngo, S. Biricik, and M. Basu, "A self-tuning grid synchronization method for active power filters," Electric Power Components and
Systems, vol. 44, no. 17, pp. 1947-1957, 2016.

[22] P. Rodríguez, J. Pou, J. Bergas, J. I. Candela, R. P. Burgos, and D. Boroyevich, "Decoupled double synchronous reference frame PLL for power converters control," IEEE Transactions on Power Electronics, vol. 22, no. 2, pp. 584-592, 2007.

[23] Z. Chedjara, A. Massoum, S. Massoum, P. Wira, A. Safa, and A. Gouichiche, "A novel robust PLL algorithm applied to the control of a shunt active power filter using a self tuning filter concept," in 2018 IEEE International Conference on Industrial Technology (ICIT). IEEE, 2018, pp. 1124-1131.

[24] M. H. Bierhoff, "A general PLL-type algorithm for speed sensorless control of electrical drives," IEEE Transactions on Industrial Electronics, vol. 64, no. 12, pp. 9253-9260, 2017.

[25] A. Bechouche, D. O. Abdeslam, T. Otmane-Cherif, and H. Seddiki, "Adaptive neural PLL for grid-connected DFIG synchronization," Jour- 
nal of Power Electronics, vol. 14, no. 3, pp. 608-620, 2014.

[26] M. E. Meral and D. Çelik, "Benchmarking simulation and theory of various PLLs produce orthogonal signals under abnormal electric grid conditions," Electrical Engineering, vol. 100, no. 3, pp. 1805-1817, 2018.

[27] E. Radwan, K. Salih, E. Awada, and M. Nour, "Modified phase locked loop for grid connected single phase inverter," International Journal of Electrical and Computer Engineering (IJECE), vol. 9, no. 5, pp. 39343943, 2019.

[28] P. Rodriguez, R. Teodorescu, I. Candela, A. V. Timbus, M. Liserre, and F. Blaabjerg, "New positive-sequence voltage detector for grid synchronization of power converters under faulty grid conditions," in 2006 37th IEEE Power Electronics Specialists Conference. IEEE, 2006, pp. $1-7$.

[29] A. K. Verma, R. K. Jarial, P. Roncero-Sanchez, M. R. Ungarala, and J. M. Guerrero, "An improved hybrid pre-filtered open-loop algorithm for three-phase grid synchronization," IEEE Transactions on Industrial Electronics, 2020.

[30] A. Safa, E. M. Berkouk, Y. Messlem, Z. Chedjara, and A. Gouichiche, "A pseudo open loop synchronization technique for heavily distorted grid voltage," Electric Power Systems Research, vol. 158, pp. 136-146, 2018.

[31] Y. Terriche, M. U. Mutarraf, M. Mehrzadi, A. Lashab, J. M. Guerrero, J. C. Vasquez, and D. Kerdoun, "Adaptive CDSC-based open-loop synchronization technique for dynamic response enhancement of active power filters," IEEE Access, vol. 7, pp. 96743-96752, 2019.

[32] I. Kamwa, M. Leclerc, and D. McNabb, "Performance of demodulationbased frequency measurement algorithms used in typical PMUs," IEEE Transactions on Power Delivery, vol. 19, no. 2, pp. 505-514, 2004.

[33] C. Marques, M. Ribeiro, and E. Da Silva, "Enhanced demodulationbased technique for estimating the parameters of fundamental component in power systems," IET generation, transmission \& distribution, vol. 5, no. 9, pp. 979-988, 2011.

[34] E. Elhoussin, V. Choqueuse, and M. E. H. Benbouzid, "Condition monitoring of induction motors based on stator currents demodulation,' International Review of Electrical Engineering, vol. 10, no. 6, pp. 704 $715,2015$.

[35] V. Choqueuse, M. E. H. Benbouzid, Y. Amirat, and S. Turri, "Diagnosis of three-phase electrical machines using multidimensional demodulation techniques," IEEE Transactions on Industrial Electronics, vol. 59, no. 4, pp. 2014-2023, 2011.

[36] K. Mathuria, I. Hussain, B. Singh, and N. Kumar, "A quadrature oscillator-based DT for accurate estimation of fundamental load current for pv system in distribution network," IEEE Transactions on Industrial Informatics, vol. 15, no. 6, pp. 3324-3333, 2018.

[37] W. C. Duesterhoeft, M. W. Schulz, and E. Clarke, "Determination of instantaneous currents and voltages by means of alpha, beta, and zero components," Transactions of the American Institute of Electrical Engineers, vol. 70, no. 2, pp. 1248-1255, 1951.

[38] H. Ahmed and M. Benbouzid, "Demodulation type single-phase PLL with DC offset rejection," Electronics Letters, vol. 56, no. 7, pp. 344 347, 2020.

[39] P. K. Meher, J. Valls, T.-B. Juang, K. Sridharan, and K. Maharatna, "50 years of CORDIC: Algorithms, architectures, and applications," IEEE Transactions on Circuits and Systems I: Regular Papers, vol. 56, no. 9, pp. 1893-1907, 2009.

[40] T. Instruments, “C28x IQmath library,” Texas Instruments, 2010.

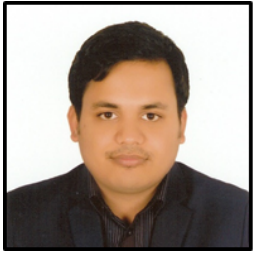

Hafiz Ahmed (S'10--M'17--SM'20) received the Ph.D. degree in Automatic Control from the University of Lille 1, France, in 2016. He received the EECI (European Embedded Control Institute) Ph.D. award in 2017 and the Best $\mathrm{PhD}$ Theses award from the Research Cluster on Modeling, Analysis and Management of Dynamic Systems (GDR-MACS) of the National Council of Scientific Research (CNRS) in France in 2017.

From 2016 to 2020, he was with Clemson University, USA, Asia Pacific University, Bangladesh, North South University, Bangladesh, and Coventry University, UK. Since 2020, he has been with Birmingham City University, UK. He is interested in applied control engineering with special focus in energy and environment. He is an Associate Editor of the INTERNATIONAL JOURNAL OF ELECTRICAL ENGINEERING \& EDUCATION.

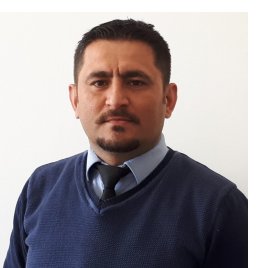

Samet Biricik (M'12--SM'19) received the B.Sc., M.Sc., and Ph.D. degrees in electrical and electronic engineering from the Near East University, Nicosia, Mersin 10, Turkey, in 2006, 2009 , and 2013, respectively. He is currently a lecturer in the European University of Lefke, Lefke and a Research Fellow in the School of Electrical and Electronic Engineering of Technological University Dublin, Dublin, Ireland. His research interests include the application of power electronics, power quality, electrical machines, and high-voltage engineering.

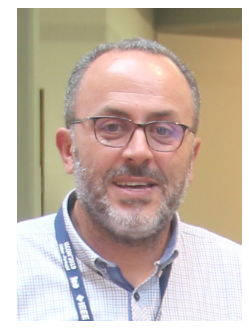

Mohamed Benbouzid (S'92--M'95--SM'98-$\left.F^{\prime} 20\right)$ received the $B . S c$. degree in electrical engineering from the University of Batna, Batna, Algeria, in 1990, the M.Sc. and Ph.D. degrees in electrical and computer engineering from the National Polytechnic Institute of Grenoble, Grenoble, France, in 1991 and 1994, respectively, and the Habilitation à Diriger des Recherches degree from the University of Picardie "Jules Verne, "Amiens, France, in 2000. After receiving the Ph.D. degree, he joined the Professional Institute of Amiens, University of Picardie "Jules Verne, "where he was an Associate Professor of electrical and computer engineering. Since September 2004, he has been with the University of Brest, Brest, France, where he is a Full Professor of electrical engineering. Prof. Benbouzid is also a Distinguished Professor and a 1000 Talent Expert at the Shanghai Maritime University, Shanghai, China. His main research interests and experience include analysis, design, and control of electric machines, variable-speed drives for traction, propulsion, and renewable energy applications, and fault diagnosis of electric machines.

Prof. Benbouzid has been elevated as an IEEE Fellow for his contributions to diagnosis and fault-tolerant control of electric machines and drives. He is also a Fellow of the IET. He is the Editor-in-Chief of the INTERNATIONAL JOURNAL ON ENERGY CONVERSION and the APPLIED SCIENCES (MDPI) Section on Electrical, Electronics and Communications Engineering. $\mathrm{He}$ is a Subject Editor for the IET RENEWABLE POWER GENERATION. He is also an Associate Editor of the IEEE TRANSACTIONS ON ENERGY CONVERSION. 